\title{
NF-кB and MAPK-Targeted Anti-Inflammatory Activity of Andrographis Paniculata Extract
}

\author{
Kwang-Soo Baek ${ }^{1}$, Jae Youl Cho ${ }^{1}$, Ji Hong Hwang', Yunyoung Kim², Hyeonjeong Choe ${ }^{2}$, Chae \\ Yun Baek ${ }^{3}$, Donghun Lee ${ }^{3}$ and Mi-Yeon Kim ${ }^{2 *}$ \\ ${ }^{1}$ Department of Integrative Biotechnology, Sungkyunkwan University, Korea \\ ${ }^{2}$ Natural F\&P Corp, Korea
}

${ }^{3}$ Department of Herbal Pharmacology, College of Korean Medicine, Gachon University, Korea

*Corresponding author: Mi-Yeon Kim, Ph.D., Director, Natural F\&P Corp, Korea

To Cite This Article: Kwang-Soo Baek, Jae Youl Cho, Ji Hong Hwang, Yunyoung Kim, Mi-Yeon Kim, et al. NF- $\kappa B$ and MAPK-Targeted Anti-Inflammatory Activity of Andrographis Paniculata Extract. Am J Biomed Sci \& Res. 2021 - 11(5). AJBSR.MS.ID.001677. DOI: 10.34297/ AJBSR.2021.11.001677.

Received: 眥 January 01, 2021; Published: 些 February 01, 2021

\begin{abstract}
Andrographis paniculata (Burm.f.) is in the Acanthaceae family. There have been reports on the various medical effects of extracts of A. paniculata and the active ingredient, andrographolide (AG). Previous study confirmed that the standardized A. paniculata extract (APE, ParActin ${ }^{\circledR}$ ) inhibited the expression of MMPs and inflammatory cytokines (IL-1 $\beta$ and IL-6) in monosodium iodoacetate (MIA)-induced cartilage degradation in rats, and relieved acetic acid-induced writhing responses in mice. To investigate the molecular actions of APE, we treated APE and AG, and examined the level of inflammatory mediators in LPS-treated RAW264.7 cells. APE and AG inhibited NO and PGE ${ }_{2}$ production in LPS-treated RAW264.7 macrophages. APE inhibited MAPK pathways (p38, JNK, and ERK) and IкB $\alpha$ phosphorylation, and increased IкB $\alpha$ degradation. The results revealed that antiinflammatory activity of APE were derived from AG by inhibition of MAPKs and NF- $\mathrm{KB}$ pathways.
\end{abstract}

Abbreviations: NO: Nitric Oxide; MAPK: Mitogen-Activated Protein Kinase; ERK: Extracellular Signal-Related Kinase; JNK: c-Jun N-Terminal

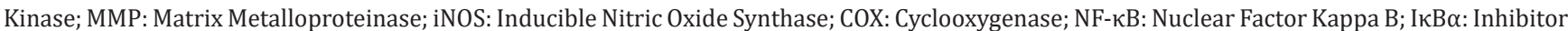
Kappa B Alpha; IKK: I Kappa B Kinase; TLR: Toll-like Receptor

\section{Introduction}

Andrographis paniculata (Burm.f.) (synonym Justicia paniculata, common name "king of bitter") is in the Acanthaceae family. A lot of research has proven that extracts of $A$. paniculata display wide spectrum of pharmacological activity such as anti-cancer $[1,2]$, anti-diarrheal [1], anti-viral [1], anti-malarial [3], anti-oxidant [4], anti-inflammatory [5], hepatoprotective [6], cardiovascular [7], and immunostimulatory activities [8].

In a prospective, randomized, and placebo-controlled trial, $A$. paniculata composition, ParActin ${ }^{\circledR}$ showed some beneficial effects on osteoarthritis patient symptoms [9]. Furthermore, treatment with A. paniculata showed a reduction in the stiffness score, fatigue, and pain in RA patients [10]. In human osteoarthritic chondrocytes, andrographolide reduces matrix metalloproteinases (MMPs) and inducible nitric oxide synthase (iNOS) [11].

There have been reports on andrographolide's antiinflammatory efficacy in several literatures. The major diterpenoid constituent of $A$. paniculata, andrographolide has received wide attention of many research groups for its diverse pharmacological properties by inhibition of NF- $\mathrm{BB}$ and MAPK pathways [12].

Previous study confirmed that the standardized A. paniculata extract (APE, ParActin ${ }^{\circledR}$ ) inhibited the expression of MMPs and inflammatory cytokines (IL-1 $\beta$ and IL-6) in monosodium iodoacetate (MIA)-induced cartilage degradation in rats, and relieved acetic acid-induced writhing responses in mice [13]. Through this study, the anti-inflammatory mechanisms of APE using LPS-treated RAW264.7 cells will be discussed further. 


\section{Materials and Methods}

\section{Materials}

High Performance Liquid Chromatography (HPLC) grade methanol and distilled water (DW) used Samchun Chemical (Incheon, Korea). Analytical standard of andrographolide and LPS (E. coli 0111:B4) were purchased from Sigma-Aldrich Chemical Co. (St. Louis, MO, USA). Fetal bovine serum (FBS) was obtained from Gibco (Grand Island, NY, USA). DMEM was purchased from Hyclone (Logan, UT, USA). RAW264.7 cells were purchased from ATCC (Rockville, MD, USA). All other chemicals were of analytical grade. Antibodies specific for either the total or phosphorylated forms of extracellular signal-related kinase (ERK), c-Jun N-terminal kinase (JNK), p38, Inhibitor kappa B alpha ( $\mathrm{I \kappa B} \alpha$ ) and $\beta$-actin were obtained from either Cell Signaling Technologies (Beverly, MA, USA) or Santa Cruz Biotechnology (Santa Cruz, CA, USA).

\section{Preparation of APE}

The leaf extracts of Andrographis paniculata Nees. (Acanthaceae) used in the experiment were purchased from HP Ingredients (Bradenton, USA). The dried leaf of $A$. paniculata was extracted in $75 \%$ ethanol solution. The extract was filtered and concentrated under decreased pressure, then, it was spray dried to yield a powder. HPLC result showed that level of andrographolide content was $53 \%$ in APE $\left(\right.$ ParActin $^{\circledR}$ ).

\section{HPLC Analysis of APE}

The chromatographic quantitative analysis, used for APE, was a HPLC analysis system, equipped with a 1200 series HPLC system (Agilent, Palo Alto, USA), as previously described [13]. The chromatic separation was accomplished on $\mathrm{C}_{18}$ column, $4.6 \times$ $150 \mathrm{~mm}, 5$ m (Shiseido capcell pak UG120, Shiseido, Yokohama, Japan) at $35^{\circ} \mathrm{C}$. The mobile phase composition was $60 \%$ of ethanol and $40 \%$ of deionized water which was set in isocratic mode and flow rate was $1 \mathrm{~mL} / \mathrm{min}$. An aliquot of $10 \mu \mathrm{L}$ of the sample was injected and the effluent was monitored at $225 \mathrm{~nm}$ and the total run time was fixed as $30 \mathrm{~min}$. Data was obtained and analyzed by Agilent Chemstation Software. A total of $1.5 \mathrm{mg}$ of standard compounds was dissolved in $1 \mathrm{~mL}$ of methanol, and then diluted to concentrations $(\mu \mathrm{g} / \mathrm{mL})$ of $12,23,47,94,188$ and 375 each. The obtained coefficients of correlation were 1.000 ( $y=12.253 x$ $+15.808)$. The contents of were quantified by comparing with the standard curve. The 100 and $600 \mathrm{mg}$ of APE were dissolved in 10 $\mathrm{mL}$ of DW and dimethyl sulfoxide (DMSO) respectively, and then sonication for $20 \mathrm{~min}$. Additionally, each sample was filtered with a $0.45 \mu \mathrm{m}$ syringe filter (Waters Corp., Milford, USA). Each sample was diluted for HPLC analysis with DW (from 60,000 to 6,000 ppm) and DMSO (from 10,000 to $100 \mathrm{ppm}$ ).

\section{Measurement of $\mathrm{NO}$ and $\mathrm{PGE}_{2}$}

RAW264.7 cells seeded at 96 well, $1 \times 10^{6} / \mathrm{ml}$, were incubated at $37^{\circ} \mathrm{C}$, and $5 \% \mathrm{CO}_{2}$ for $24 \mathrm{~h}$. Cells were treated with different concentrations of APE or AG $(0.5-16 \mu \mathrm{g} / \mathrm{mL})$ and LPS $(1 \mu \mathrm{g} / \mathrm{mL})$ and incubated for $24 \mathrm{~h}$. The inhibitory effect of APE on NO and $\mathrm{PGE}_{2}$ production was determined by analysis of $\mathrm{NO}$ and $\mathrm{PGE}_{2}$, using Griess reagent and an enzyme-linked immunosorbent assay kit (R\&D systems, Minneapolis, MN, USA), as described previously with some modification [14].

\section{Cytotoxicity}

The cytotoxic effects of APE or AG were then evaluated using a conventional MTT assay, as reported previously with some modification [14]. After $24 \mathrm{~h}$ of seeding, $5 \mathrm{mg} / \mathrm{mL}$ MTT solution was added to RAW264.7 cells and kept at $37^{\circ} \mathrm{C}, 5 \% \mathrm{CO}_{2}$ for $1 \mathrm{~h}$. The supernatant was removed, $200 \mu \mathrm{L}$ of DMSO was added, and the absorbance was measured at $550 \mathrm{~nm}$.

\section{Western Blot Analysis}

The protein expression of the total or phosphorylated forms of p38, ERK, JNK, I $\mathrm{KB} \alpha$, and $\beta$-actin was analyzed through Western blot analysis. RAW264.7 cells seeded at 6 well, $1 \times 10^{6} / \mathrm{ml}$, were incubated at $37^{\circ} \mathrm{C}$, and $5 \% \mathrm{CO}_{2}$ for $24 \mathrm{~h}$. Cells were treated with different concentrations of APE, $2 \mu \mathrm{g} / \mathrm{mL}$ and LPS $1 \mu \mathrm{g} / \mathrm{mL}$, and incubated for indicated time. The intracellular protein was extracted by RIPA buffer. The protein concentration is measured by bradford assay. The extracted protein was denatured by LDS sample buffer (Thermo Scientific, Massachusetts, USA).

Equal amounts of protein samples were loaded onto SDSpolyacrylamide gel for electrophoresis and the isolated proteins were transferred to PVDF membranes with the Semidry Transfer Cell (Bio-Rad Laboratories, Inc., California, USA) for $7 \mathrm{~min}$ at 25 V. Membranes were incubated at room temperature with $3 \%$ BSA in TBS-T for $1 \mathrm{~h}$ to block non-specific antibody binding. After that, membrane was washed three times with TBS-T and primary antibodies (p38, ERK, JNK, I $\mathrm{B} \alpha$, and $\beta$-actin) were applied to react for $15 \mathrm{~h}$ at $4^{\circ} \mathrm{C}$. The antibodies were purchased from Cell signaling technology, and Santa Cruz Biotechnology, Inc. The membrane was probed using secondary antibody at room temperature for $1 \mathrm{~h}$ and then reacted with D-Plus ${ }^{\mathrm{TM}}$ ECL Femto System (Dongin, Seoul, Korea) solution. The Western blot image was identified with LAS3000 (Fujifilm, Tokyo, Japan). The blot bend densities show mean \pm SEM values of three different independent experiments [15].

\section{Statistics}

The statistical significance of differences between the control and experimental groups was performed using Student's $t$-test. The 
significance was verified at $P<0.05$ level, and measurements were indicated as mean \pm standard error of the mean.

\section{Results and Discussion}

\section{Analysis of andrographolide in DW, DMSO solution of the APE and AG standard by HPLC}

As shown in Figure 1, it was confirmed that the $6,000 \mathrm{ppm}$ DW and 100 ppm DMSO solution of the APE contained 17.2 and
58.0 ppm AG, respectively. As in a previous study [16], The low solubility of $A G$ on the water is a major limitation of research in cell culture. This limitation shows that the use of organic solvents such as ethanol and DMSO can be an alternative to AG dissolution. DMSO solution of APE was 3.4 times higher in AG content than DW solution. This result suggests that DMSO solution was the optimal extraction solvent. Since APE contains about $50 \%$ of AG content, the experiment was carried out by preparing the concentration of APE $(1-16 \mu \mathrm{g} / \mathrm{mL})$ and $\mathrm{AG}(0.5-8 \mu \mathrm{g} / \mathrm{mL})$ to be twice.

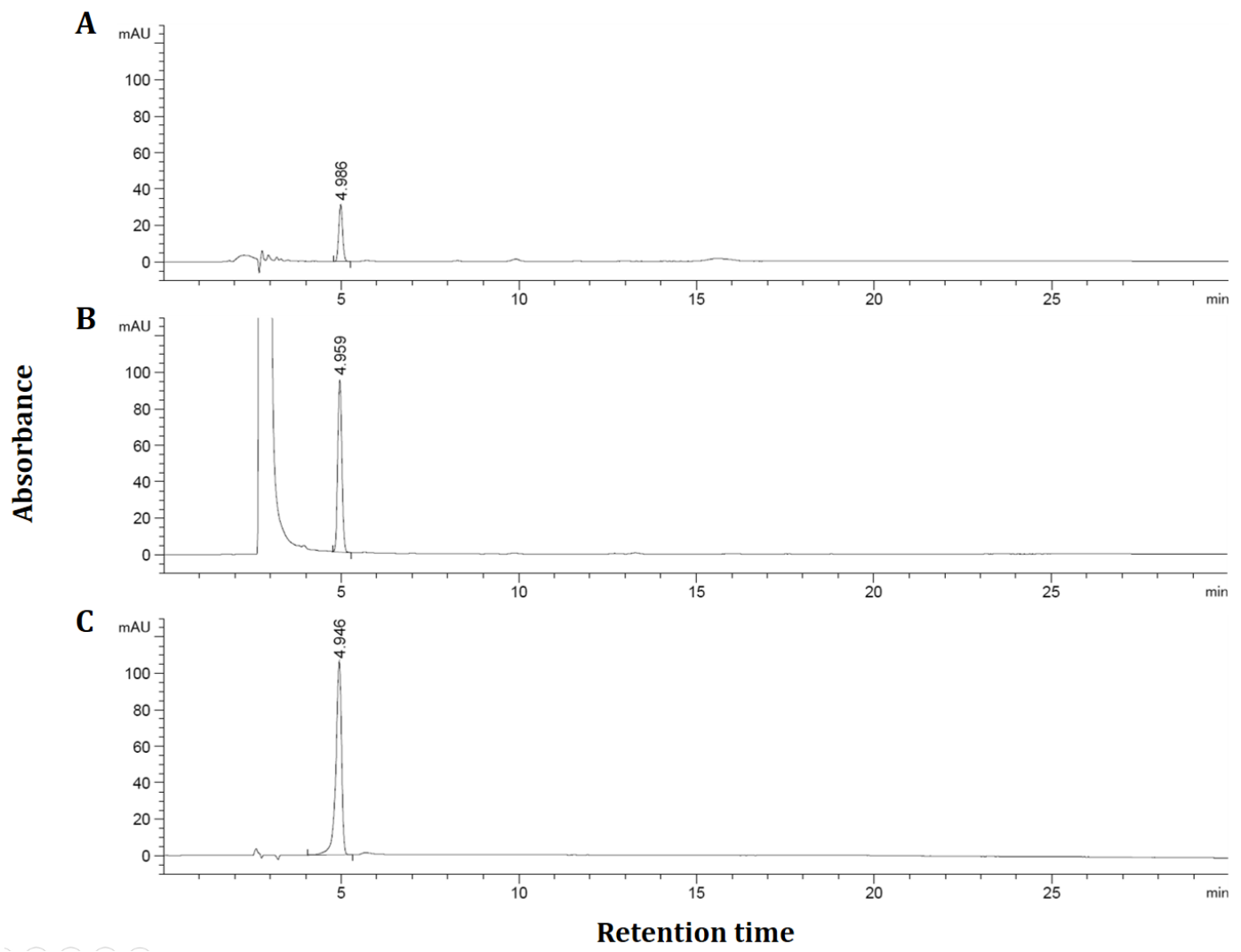

Figure1: HPLC chromatogram of APE re-dissolved in (A)DW, (B) DMSO, (C) standard andrographolide.

\section{Production of Nitric oxide in Macrophages}

Nitric oxide (NO) is an important regulatory and effector molecule with various biological functions. [17]. Once iNOS is induced, it produces large amount of NO that profoundly influences and damages cell and tissue function. Large quantity of NO produced by LPS stimulation might play a critical role in LPSinduced tissue damage. NO is a pivotal inflammatory factor which contributes to OA progression by modulation of pro-inflammatory or pro-catabolic factors, including Nos2, Il1b, Mmp13 and Ptgs2. OA chondrocytes overexpress iNOS and its product, NO, which has been considered as a biomarker for OA [18].

Previous studies have shown that AG inhibits NO production in various in vitro and in vivo models $[18,19]$. In LPS-induced RAW264.7 cells, APE suppressed the level of NO. APE dissolved in DMSO at different concentrations, the NO production of LPSinduced RAW264.7 cells was dose-dependently decreased by APE at 1-16 $\mu \mathrm{g} / \mathrm{mL}\left(\mathrm{IC}_{50} 5.3 \mu \mathrm{g} / \mathrm{mL}\right.$ ) (Figure $2 \mathrm{~A}$ ). In LPS-induced RAW264.7 cells, AG suppressed the level of NO in a dose-dependent manner ( $\mathrm{IC}_{50} 3.1 \mu \mathrm{g} / \mathrm{mL}$ ) (Figure 2B). This is because the content of AG contained in APE is $50 \%$, which is similar to $\mathrm{IC}_{50}$, so $\mathrm{AG}$ is predicted to be a major active functional ingredient showing the anti-inflammatory effect of APE. The results of this study are consistent with the results of a study in which AG inhibited iNOS gene expression and NO production in a LPS-induced RAW264.7 cells $[20,21]$. 

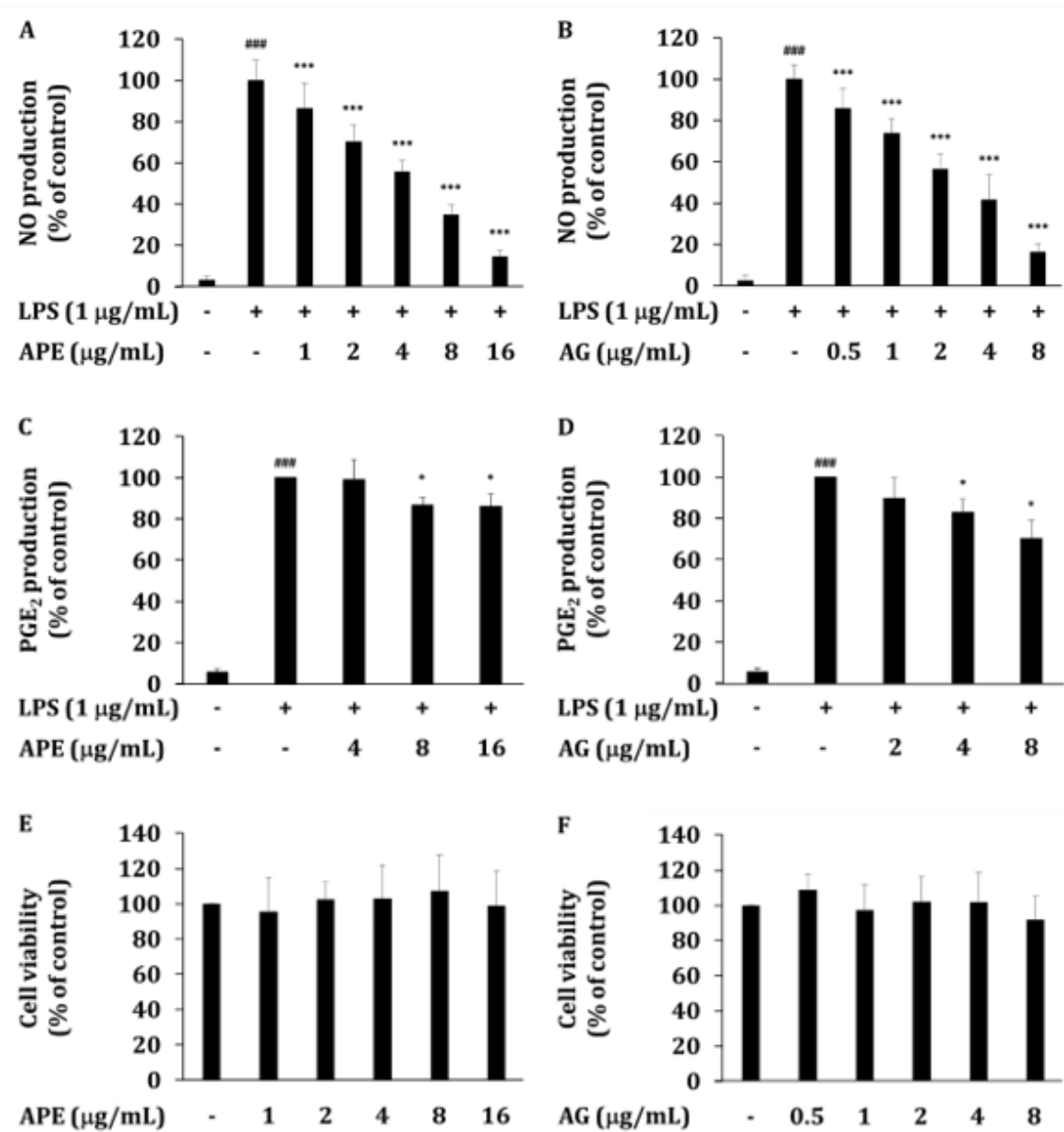

Figure 2: The inhibitory effect of APE or AG on production of inflammatory mediators. $(A, B)$ Levels of $N O$, and $(C, D) P G E_{2}$ were determined in culture supernatants of RAW264.7 cells treated with APE or AG with LPS $(1 \mu \mathrm{g} / \mathrm{mL})$ for $24 \mathrm{~h}$. (E, F) The viability of RAW264.7 cells was determined by MTT assay. Data are presented as the mean $\pm \mathrm{SD}$ of an experiment done with 6 biological replicates $(n=6)$ per treatment. $* P<0.05, * * P<0.01$ and $* * * P<0.001$ compared to LPS treated group. \#\#P $<0.001$ compared to LPS non-treated group.

Our previous study [13], the efficacy was also confirmed in the results of suppressing iNOS expression and NO production in the LPS induction model using APE - water soluble fraction dissolved in water. Through this, it is expected that the inhibitory effect of APE on NO production in this study was revealed by AG, which is contained in APE, suppressing the gene expression of iNOS. Also, inhibition of NO production by AG was confirmed. This result suggested that APE could be a useful complement in the treatment of arthritis symptoms $[9,10]$.

\section{Production of $\mathrm{PGE}_{2}$ in Macrophages}

$\mathrm{PGE}_{2}$ is synthesized from arachidonic acid by COX-2 and overexpressed after being stimulated by LPS, resulting in sepsisrelated inflammatory symptoms and signs [22]. In addition, $\mathrm{PGE}_{2}$ has been reported to play a role in releasing pro-inflammatory cytokines, such as TNF- $\alpha$ and IL-6 in many cells [23]. Pain is perceived through activation of endings of nociceptive afferent nerves by pain-producing substances, released from tissue, sensitized by locally produced prostaglandins [24]. Because AG has been reported to have an inhibitory effect on $\mathrm{PGE}_{2}$ production $[5,19,20]$, PGE $_{2}$ expression in LPS-induced RAW264.7 cells was observed.

In LPS-induced RAW264.7 cells, APE suppressed the level of $\mathrm{PGE}_{2}$. The PGE $\mathrm{P}_{2}$ production of LPS-induced RAW264.7 cells was dose-dependently decreased by APE at $4-16 \mu \mathrm{g} / \mathrm{mL}$ (Figure $2 \mathrm{C}$ ). AG prepared with the concentration of AG contained in APE showed an equivalent inhibitory effect in LPS-induced RAW264.7 cells (Figure 2D).

Our previous study [13], APE administration resulted the analgesic effects on acetic acid-induced writhing responses. This result shows the analgesic effects of APE against peripheral $\mathrm{PGE}_{2}$ synthesis by AG. It was confirmed that APE inhibited $\mathrm{PGE}_{2}$ production by AG. This result suggested that APE could be a useful complement in the treatment of arthritis symptoms $[9,10]$.

\section{Activation of MAPKs in Macrophages}

LPS treatment in macrophages is well known to activate the MAPK pathway through TLR4. MAPKs regulate the production of 
pro-inflammatory cytokines and enzymes such as IL-1 $\beta$, TNF- $\alpha$, and IL-6, iNOS COX-2. Previous studies have shown that AG inhibits $\mathrm{NO}, \mathrm{PGE}_{2}$ production and MAPKs activity in various in vitro/in vivo inflammation models [5].

Because the production of $\mathrm{NO}$ and $\mathrm{PGE}_{2}$ were mainly regulated by MAPKs, we investigated the possible involvement of p38, ERK, and JNK in APE inhibition. Before extracting whole lysates, NO concentration was measured and NO decreases by about $90 \%$ after $24 \mathrm{~h}$ of APE $2 \mu \mathrm{g} / \mathrm{mL}$ treatment in 6 wells (data not shown). To determine the inhibitory effects of APE on LPS-activated MAPKs activities, total lysates prepared from RAW264.7 cells treated with LPS for each 15, 30, and 45 min were incubated with APE. Phosphorylation of $\mathrm{p} 38$ by LPS was significantly inhibited by $74 \%$ $(P<0.01)$ or $93 \%(P<0.001)$ at concentrations of $2 \mu \mathrm{g} / \mathrm{mL}$ of APE for $15 \mathrm{~min}$ and $30 \mathrm{~min}$, respectively (Figure 3A-B). In addition, phosphorylation of ERK was significantly inhibited by $9 \%(P<0.01)$ and $64 \%(P<0.05)$ at 30 and 45 min treatment, respectively (Figure 3C). Finally, the phosphorylation of JNK was significantly inhibited by $61 \%(p<0.001)$ at 45 min treatment (Figure 3D). These results showed a similar pattern to the results of inhibiting the phosphorylation of MAPKs, which was treated with LPS for 15 mins when $\mathrm{AG}$ was pretreated at concentrations of $1 \mu \mathrm{M}$ and $10 \mu \mathrm{M}[21]$.

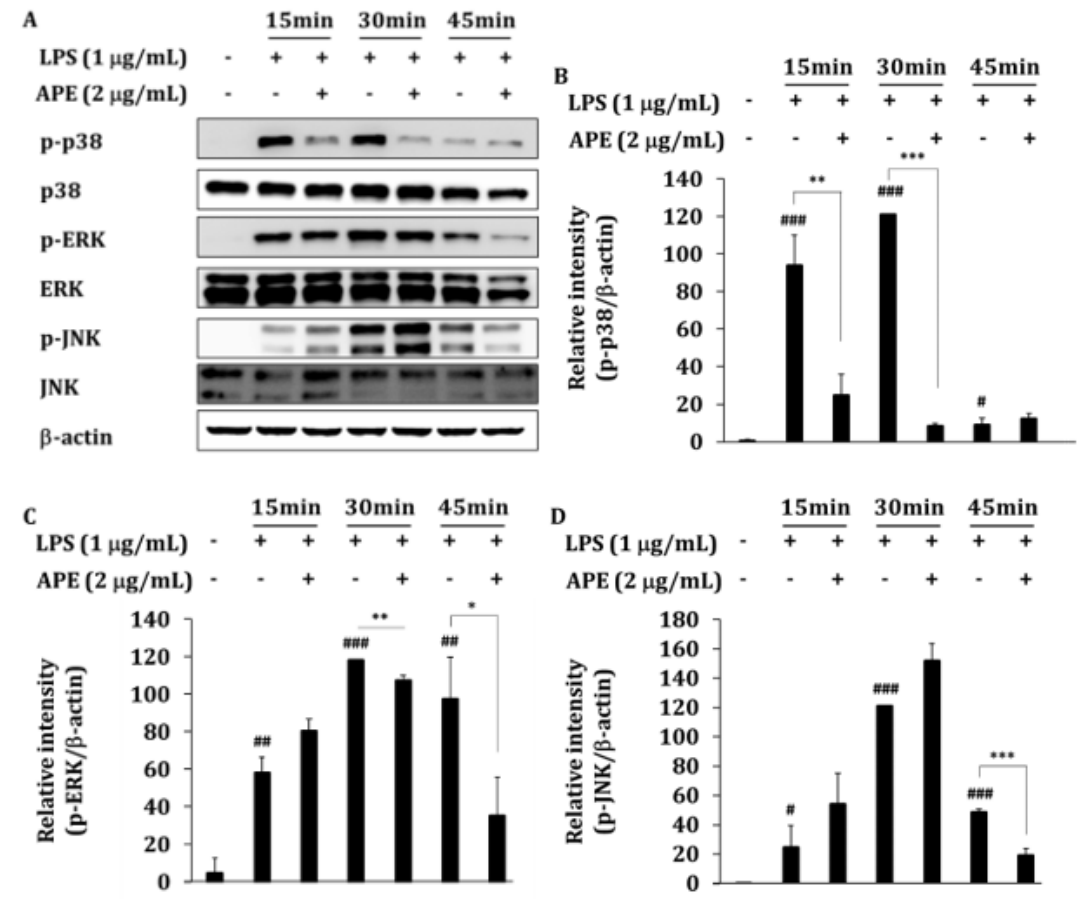

Figure 3: The inhibitory effect of APE on MAPKs activation. (A) RAW264.7 cells $\left(1 \times 10^{6}\right.$ cells $\left./ \mathrm{mL}\right)$ were incubated with APE in the absence or presence of LPS $(1 \mu \mathrm{g} / \mathrm{mL})$ for the indicated times. After preparing whole cell lysates, levels of total or phospho-forms of upstream signaling enzymes (ERK, p38, and JNK) were identified by immunoblotting. Other data are representative images of three different experiments that had similar results. (B-D) The blot bend densities show mean \pm SEM values of three different independent experiments. $* P<0.05, * * P<0.01$ and *** $P<0.001$ APE treated vs. APE non-treated LPS treated group. \#P<0.05, \#\#P<0.01 and \#\#P<0.001 compared to LPS non-treated group.

Through this, it was confirmed that the extract of APE inhibits the activation of the MAPK pathway. In our previous study [13], APE inhibited the expression of iNOS, COX-2 in an in vitro LPS model, and pro-inflammatory cytokines IL- $1 \beta$, TNF- $\alpha$, and IL- 6 in the blood in rat osteoarthritis (OA) model. Therefore, the MAPK mechanism affects joint damage and bone damage caused by joint inflammation, so it is expected to have a pain relief effect in the arthritis clinical trial of APE $[9,10]$.

\section{Activation of NF- $\kappa B$ in Macrophages}

The transcription factor NF- $\mathrm{kB}$ is inactivated in the form of a complex with the inhibitory protein $I \kappa B \alpha$ and is present in the cytoplasm. LPS inflammatory stimulation activates I kappa B kinase (IKK) and thereby phosphorylates I $\mathrm{B} \alpha$. Phosphorylation of I $\mathrm{B} \alpha$ induces ubiquitination, thereby promoting its degradation by the

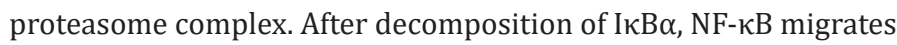
to the nucleus, increasing the expression of inflammatory mediator genes such as iNOS and COX-2 and pro-inflammatory cytokines, IL$1 \beta$, IL-6, and TNF- $\alpha$ [25]. MMPs are synthesized and secreted by chondrocytes in response to stimulants including IL-1 and TNF. Most evidence suggests that matrix degradation is achieved through the action of MMPs (MMP-1, 3, 8, 13). In OA, the degradation of extracellular matrix is driven mainly by these enzymes from the chondrocytes [26]. 
Previous report has shown that AG inhibited NF- $\mathrm{KB}$ and the production of MMP-1, 3, 13 in IL-1 $\beta$-treated cartilage-derived chondrocytes of osteoarthritis patients [11]. Similarly to this report, several kinds of $A$. paniculata extracts also inhibited NF- $\kappa B$ pathway in in vivo models $[27,28]$.

To determine the inhibitory effects of APE on LPS-activated NF$\kappa \mathrm{B}$ activities, total lysates prepared from RAW264.7 cells treated with LPS for 15, 30, and 45 min were incubated with APE (Figure $4 \mathrm{~A})$. The phosphorylation of $\mathrm{I} \kappa \mathrm{B} \alpha$ significantly inhibited by $96 \%$ $(P<0.01)$ at $15 \mathrm{~min}$, and $35 \%(P<0.05)$ at $45 \mathrm{~min}$ after exposure of the cells to APE (Figure 4B). Due to the suppression of the phosphorylation of $I \kappa B \alpha$, the degradation of $I \kappa B \alpha$ was suppressed at $30 \mathrm{~min}$. The amount of I $\mathrm{B} \alpha \alpha$ proteins increased significantly by $131 \%(p<0.05)$ (Figure 4C).
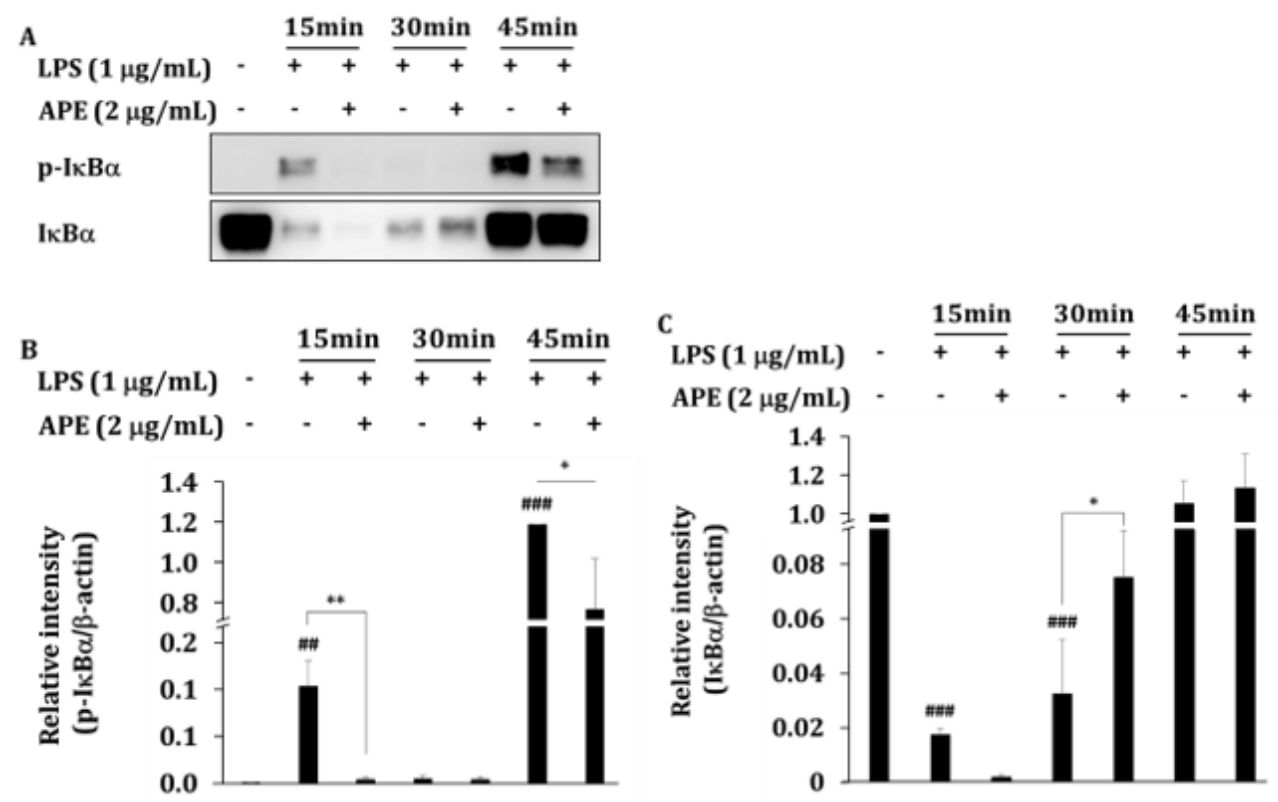

Figure 4: The inhibitory effect of APE on IKBa activation. (A) RAW264.7 cells $\left(1 \times 10^{6} \mathrm{cells} / \mathrm{mL}\right)$ were incubated with APE in the absence or presence of LPS $(1 \mu \mathrm{g} / \mathrm{mL})$ for the indicated times. After preparing whole cell lysates, levels of total or phospho-form of upstream signalling enzyme, IKBa was identified by immunoblotting. Other data are representative images of three different experiments that had similar results. (B, C) The blot bend densities show mean \pm SEM values of three different independent experiments. $* P<0.05, * * P<0.01$ APE treated vs. APE non-treated control group. \#\#P<0.01 and \#\#\# $<0.001$ compared to LPS non-treated group.

The results are similar to the previous study showing that phosphorylation of I $\mathrm{B} \alpha$ was significantly inhibited when $30 \mu \mathrm{g} /$ $\mathrm{mL}$ of $A$. paniculata methanol extract was pretreated for $1 \mathrm{~h}$ and then treated with LPS for $15 \mathrm{~min}$ [29]. In this study, APE $2 \mu \mathrm{g} / \mathrm{mL}$ was treated at the same time as LPS, and the same effect was shown after $15 \mathrm{~min}$.

In this study, it was confirmed by Western blot that

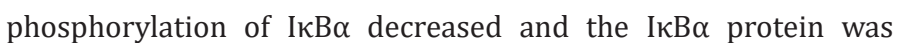
degraded by APE. Results are consistent with a mechanism of APE that inhibits the production of IL- $1 \beta$, IL-6, and TNF- $\alpha$, proinflammatory cytokines produced by NF- $\kappa B$ gene expression in rat osteoarthritis models and in vitro LPS models. These results point to APE as the anti-inflammatory ingredient that inhibits the expression of iNOS and COX-2 genes [13].

In addition to APE, it supports the effect of protecting joint cartilage by inhibiting MMP-1, 3, 8, 13 in rat osteoarthritis model. Therefore, it affects joint damage and joint cartilage decomposition due to joint inflammation through the NF- $\kappa B$ mechanism. It is expected to alleviate arthritis symptoms in the arthritis clinical trial $[9,10]$.

\section{Conclusion}

The study demonstrated that APE suppresses in vitro inflammatory responses in LPS-TLR4-activated macrophages. APE inhibited the expression of NO and $\mathrm{PGE}_{2}$ increased by LPS in a dose-dependent manner. APE acts as the inhibitor of MAPKs (p38, ERK and JNK) and I $\mathrm{B} \alpha$ which is particularly important in iNOS/ COX-2 expression. AG-containing Andrographis paniculata has been ethnopharmacologically used for a long time and is orally available $[18,24]$. Based on the results of the previous clinical trials and toxicity tests on A. paniculata, it is expected to be used as a new antiinflammatory material as a substitute for various inflammatory disease treatments, such as osteoarthritis.

\section{Conflict of Interest}

The authors declare no conflict of interest. 


\section{References}

1. Biswa Deepak Bharati, Pramod Kumar Sharma, Nitin Kumar, Rupesh Dudhe, Vipin Bansal, et al. (2011) Pharmacological Activity of Andrographis paniculata: A Brief Review. Pharmacologyonline 2: 1-10.

2. Takakuni Matsuda, Masanori Kuroyanagi, Satooko Sugiyama, Kaoru Umehara, Akira Ueno, et al. (1994) Cell differentiation-inducing diterpenes from Andrographis paniculata Nees. Chem Pharm Bull 42(6): 1216-1225.

3. Nik Najib Nik A Rahman, Takahisa Furuta, Somei kojima, Kikuchi Takane, Mustafa Ali Mohd, et al. (1999) Antimalarial activity of extracts of Malaysian medicinal plants. J Ethnopharmacol 64(3): 249-254.

4. K Sheeja, P K Shihab, G Kuttan (2006) Antioxidant and anti-inflammatory activities of the plant Andrographis paniculata Nees. Immunopharmacol Immunotoxicol 28(1): 129-140.

5. Yu Li, Shengnan He, Jishun Tang, Nana Ding, Xiaoyan Chu, et al. (2017) Andrographolide Inhibits Inflammatory Cytokines Secretion in LPSStimulated RAW264.7 Cells through Suppression of NF- $\kappa$ B/MAPK Signaling Pathway. Evid Based Complement Alternat Med 2017: 8248142 .

6. R Nagalekshmi, Aditya Menon, Dhanya K Chandrasekharan, Cherupally Krishnan Krishnan Nair, et al. (2011) Hepatoprotective activity of Andrographis paniculata and Swertia chirayita. Food Chem Toxicol 49(12): 3367-3373.

7. C Y Zhang, B K H Tan (1997) Mechanisms of cardiovascular activity of Andrographis paniculata in the anaesthetized rat. J Ethnopharmacol 56(2): 97-101.

8. Mirentxu I Iruretagoyena, Jaime A Tobar, Pablo A González, Sofía E Sepúlveda, Claudio A Figueroa, et al. (2005) Andrographolide interferes with $\mathrm{T}$ cell activation and reduces experimental autoimmune encephalomyelitis in the mouse. J Pharmacol Exp Ther 312(1): 366-372.

9. Juan L Hancke, Shalini Srivastav, Dante D Cáceres, Rafael A Burgos (2019) A double-blind, randomized, placebo-controlled study to assess the efficacy of Andrographis paniculata standardized extract (ParActin $®$ ) on pain reduction in subjects with knee osteoarthritis. Phytother Res 33(5): 1469-1479.

10. R A Burgos, J L Hancke, J C Bertoglio, V Aguirre, S Arriagada, et al. (2009) Efficacy of an Andrographis paniculata composition for the relief of rheumatoid arthritis symptoms: a prospective randomized placebocontrolled trial. Clin Rheumatol 28(8): 931-946.

11. Qian hai Ding, Xiao wei Ji, Ye Cheng, Yuan quan Yu, Yi ying Qi, et al. (2013) Inhibition of matrix metalloproteinases and inducible nitric oxide synthase by andrographolide in human osteoarthritic chondrocytes. Mod Rheumatol 23(6): 1124-1132.

12. R N Charkravarti, D Charkravarti (1951) Andrographolide, the active constituent of Andrographis paniculata nees; a preliminary communication. Ind Med Gaz 86(3): 96-97.

13. Donghun Lee, Chae Yun Baek, Ji Hong Hwang, Mi Yeon Kim (2020) Andrographis paniculata Extract Relieves Pain and Inflammation in Monosodium Iodoacetate-Induced Osteoarthritis and Acetic AcidInduced Writhing in Animal Models. Processes 8(873): 1-17.

14. Deok Jeong, Young Su Yi, Gi Ho Sung, Woo Seok Yang, Jae Gwang Park, et al. (2014) Anti-inflammatory activities and mechanisms of Artemisia asiatica ethanol extract. J Ethnopharmacol 152(3): 487-496.
15. Jianjun $\mathrm{Xu}$, Yongxin Zhao, Haji Akber Aisa (2017) Anti-inflammatory effect of pomegranate flower in lipopolysaccharide (LPS)-stimulated RAW264.7 macrophages. Pharm Biol 55(1): 2095-2101.

16. Aalok Basu, Sujay Guti, Sonia Kundu, Aatrayee Das, Suvadra Das, et al. (2020) Oral andrographolide nanocrystals protect liver from paracetamol induced injury in mice. J Drug Deliv Sci Technol 55(101406).

17. Klaus D Kröncke, Karin Fehsel, Victoria Kolb Bachofen (1997) Nitric oxide: cytotoxicity versus cytoprotection - how, why, when and where? Nitric Oxide Biol Chem 1(2): 107-120.

18. Pan Jin, Christian Wiraja, Jinmin Zhao, Jinlu Zhang, Li Zheng, et al. (2017) Nitric oxide nanosensors for predicting the development of osteoarthritis in rat model. ACS Appl Mater Interfaces 9(30): 2512825137.

19. C V Chandrasekaran, Anumita Gupta, Amit Agarwal (2010) Effect of an extract of Andrographis paniculata leaves on inflammatory and allergic mediators in vitro. J Ethnopharmacol 129(2): 203-207.

20. Ting Shen, Woo Seok Yang, Young Su Yi, Gi Ho Sung, Man Hee Rhee, et al. (2013) AP-1/IRF-3 Targeted Anti-Inflammatory Activity of Andrographolide Isolated from Andrographis paniculata. Evid Based Complement Alternat Med 2013: Pp 210736.

21. Nami Kima, Peeraphong Lertnimitphuna, Yiwen Jianga, Hongsheng Tana, Hua Zhou, et al. (2019) Andrographolide inhibits inflammatory responses in LPS-stimulated macrophages and murine acute colitis through activating AMPK. Biochem Pharmacol 170: Pp 113646.

22. Jessica K Norberg, Earlphia Sells, Hui Hua Chang, Srinivas R Alla, Shuxing Zhang, et al. (2013) Targeting inflammation: multiple innovative ways to reduce prostaglandin E2. Pharm Pat Anal 2(2): 265-288.

23. Joy A. Williams, Emily Shacter (1997) Regulation of macrophage cytokine production by prostaglandin E2. Distinct roles of cyclooxygenase-1 and -2. J Biol Chem 272(41): 25693-25699.

24. Regina M Botting, Jack H Botting (2000) Pathogenesis and Mechanisms of Inflammation and Pain, Clin Drug Investig 19: 1-7.

25. Vito J Palombella, Oliver J Rando, Alfred L Goldbergt, Tom Maniatis (1994) The ubiquitin-proteasome pathway is required for processing the NF- kappa B1 precursor protein and the activation of NF- kappa B. Cell 78(5): 773-785.

26. Yin Song Wu, Yun Yu Hu, Rui Fu Yang, Zhen Wang, Yi Yong Wei, et al. (2007) The matrix metalloproteinases as pharmacological target in osteoarthritis: statins may be of therapeutic benefit. Med Hypotheses 69(3): 557-559.

27. Wei Zou, Zuoqi Xiao, Xiaoke Wen, Jieying Luo, Shuqiong Chen, et al. (2016) The anti-inflammatory effect of Andrographis paniculata (Burm. f.) Nees on pelvic inflammatory disease in rats through down-regulation of the NF- $\kappa$ B pathway. BMC Complement Altern Med 16(1): Pp 483.

28. Wen Wan Chao, Yueh Hsiung Kuo, Shie Liang Hsieh, Bi Fong Lin (2011) Inhibitory Effects of Ethyl Acetate Extract of Andrographis paniculata on NF- $\kappa B$ Trans-Activation Activity and LPS-Induced Acute Inflammation in Mice. Evid Based Complement Alternat Med 2011: Pp 254531.

29. Ji Yun Jung, Chung A Park (2020) Anti-inflammatory Efects of Andrographis Herba MeOH Extract on LPS-induced Raw 264.7 Cells. Herbal Formula Science 28(2): 147-155. 